\title{
Pain behavior mediates the relationship between perceived injustice and opioid prescription for chronic pain: a Collaborative Health Outcomes Information Registry study
}

\author{
This article was published in the following Dove Press journal: \\ Journal of Pain Research \\ 7 March 2017 \\ Number of times this article has been viewed
}

\author{
Junie S Carriere' \\ Marc-Olivier Martel ${ }^{2,3}$ \\ Ming-Chih $\mathrm{KaO}^{4}$ \\ Michael JL Sullivan ${ }^{5}$ \\ Beth D Darnall ${ }^{4}$ \\ 'Department of Psychology, ${ }^{2}$ Faculty \\ of Dentistry, ${ }^{3}$ Department of \\ Anesthesia, Faculty of Medicine, \\ McGill University, Montreal, QC, \\ Canada; ${ }^{4}$ Division of Pain Medicine, \\ Stanford Systems Neuroscience and \\ Pain Laboratory, Department of \\ Anesthesiology, Perioperative and Pain \\ Medicine, Stanford University School \\ of Medicine, Palo Alto, CA, USA; \\ ${ }^{5}$ Faculty of Health and Behavioural \\ Sciences, University of Queensland, \\ Herston, QLD, Australia
}

Background and purpose: Perceived injustice has been defined as an appraisal regarding the severity and irreparability of loss associated with pain, blame and a sense of unfairness. Recent findings have identified perceived injustice as an important risk factor for pain-related outcomes. Studies suggest that perceived injustice is associated with opioid prescription in patients with pain conditions. However, the mechanisms by which perceived injustice is linked to opioid prescription are not well understood. The primary objective of this study was to examine the potential mediating roles of pain intensity, depressive symptoms and pain behavior in the association between perceived injustice and opioid prescription among patients with chronic pain. Methods: This cross-sectional study used a sample of 344 patients with chronic pain being treated at a tertiary pain treatment center. Participants completed measures of perceived injustice, pain intensity, depressive symptoms, pain behavior and opioid prescription. Bootstrapped multiple mediation analyses were used to examine the mediating role of patients' pain intensity, depressive symptoms and pain behavior in the association between perceived injustice and opioid prescription.

Results: Consistent with previous research, we found a significant association between perceived injustice and opioid prescription. Interestingly, results revealed that pain behavior was the only variable that mediated the association between perceived injustice and opioid prescription.

Conclusion: This study was the first to examine the mechanisms by which perceived injustice is associated with opioid prescription in patients with chronic pain. We found that pain behavior, rather than pain intensity and depressive symptoms, mediated the association between perceived injustice and opioid prescription. Future research in this area should employ a longitudinal research design in order to arrive at clearer causal conclusions about the relationships between pain behavior, perceived injustice and opioid prescription.

Keywords: perceived injustice, pain intensity, depression, pain behavior, opioids, chronic pain, CHOIR

\section{Introduction}

The experience of chronic pain is a likely context within which individuals may experience perceived injustice. Perceived injustice has been defined as an appraisal regarding the severity and irreparability of loss associated with pain, as well as thoughts of blame and unfairness. Themes of injustice are often reflected in patients' statements such as "it all seems so unfair", "nothing will ever be the same" and "I can't believe this happened to me". ${ }^{1}$ Accumulating evidence suggests that perceived injustice is a
Correspondence: Junie S Carriere Department of Psychology, McGill University, 1205 Dr. Penfield Avenue, Montreal, QC H3A IBI, Canada

$\mathrm{Tel}+\mathrm{I} 5143986127$

Fax +I 5143984896

Email junie.carriere@mail.mcgill.ca 
risk factor for adverse pain-related outcomes. In fact, studies have shown that perceived injustice is associated with greater pain severity, depressive symptoms, post-traumatic stress symptoms, heightened pain behaviors, disability and lower probability of returning to work. ${ }^{2}$

Recent research has pointed to an association between perceptions of injustice and medication use. For example, in one study, perceptions of injustice were associated with increased likelihood of using pain medications in patients reporting musculoskeletal pain. ${ }^{3}$ In a study examining the association between perceived injustice and opioid prescription following whiplash injury, findings revealed that high perceived injustice prospectively predicted opioid use at 1-year follow-up. ${ }^{4}$ Little is currently known about the mechanisms linking perceived injustice to opioid prescription.

One possibility is that perceived injustice might contribute to higher levels of pain, in turn, increasing the risk of opioid prescription. In other words, pain reports of individuals who are high in perceived injustice might lead prescribers to consider that a prescription of a strong analgesic is indicated. It has also been suggested that perceptions of injustice might augment the emotional impact of negative events and lead to symptoms of depression. ${ }^{5,6}$ Accumulating evidence suggests that perceived injustice is associated with heightened levels of depressive symptoms among individuals with persistent musculoskeletal pain, injury-related pain and fibromyalgia. ${ }^{1,6-11}$ Studies have also shown that patients with depression are more likely to receive opioid prescriptions for chronic pain. ${ }^{12-14}$ Research that has been conducted to date suggests that depressive symptoms are associated with increased rates of psychological and physical symptoms and that pain arising from chronic health conditions is also rated as more severe in the presence of depressive symptoms. ${ }^{15,16}$ One intriguing possibility is that opioids may be prescribed to treat a poorly differentiated state of mental and physical pain in patients with high injustice perceptions and concurrent symptoms of depression. Because of these concerns, many investigators have turned their attention to psychological determinants of opioid prescription for chronic pain.

There are also indications that individuals who are high in perceived injustice have a propensity to use pain behavior to emphasize the magnitude of their suffering. ${ }^{17,18}$ Pain behavior refers to movement alterations or expressive displays, such as holding or rubbing affected areas of the body, activity avoidance, facial grimaces and vocalizations. ${ }^{19}$ The observation of heightened pain behavior might lead prescribers to infer high levels of pain and disability and, in turn, consider more aggressive medical pain management. Indeed, research has demonstrated that clinicians are more likely to prescribe opioids for chronic pain in patients who display more pain behavior. ${ }^{20}$ Given their association with perceived injustice and opioid prescription, it is possible that pain intensity, depressive symptoms and/or pain behavior may be pathways by which perceived injustice leads to opioid prescription for chronic pain.

The use of opioid therapy for chronic pain has increased dramatically in the past two decades. Despite the potential benefits, a significant percentage of chronic pain patients receiving opioid therapy will misuse their medication. ${ }^{12,21-25}$ This presents a significant challenge to prescribers trying to manage chronic pain while mitigating the risk for adverse opioid-related outcomes (e.g., opioid misuse, opioid abuse, worsening pain, altered hormones and accidental drug overdose). The ability to identify patients at greater risk for opioid prescription may help reduce opioid prescriptions and mitigate risk for adverse outcomes. Therefore, the purpose of this study was to examine the potential roles of pain intensity, depressive symptoms and pain behavior as mediators of the association between perceived injustice and opioid prescription. It was hypothesized that the association between perceived injustice and opioid prescription would be mediated by pain intensity, depressive symptoms and pain behavior.

\section{Methods}

\section{Participants}

Data were collected from 344 patients who presented for initial medical evaluation at the Stanford Outpatient Pain Management Center between April and July 2015. The Pain Management Center at Stanford University Medical Center offers a comprehensive range of services for patients dealing with acute or chronic pain problems. An interdisciplinary team of specialists composed of anesthesiologists, neurologists, surgeons, psychologists, physical and occupational therapists and nurse practitioners work together to address patients' pain and design patient-tailored treatment plans. The study sample included all patients who completed measures of perceived injustice, pain intensity, depressive symptoms, pain behavior and opioid prescription.

\section{Procedures}

Data were collected using the Pain Collaborative Health Outcomes Information Registry (CHOIR) ${ }^{26}$ (http://snapl. stanford.edu/choir). CHOIR is an open source platform for a health registry and learning health system. The PatientReported Outcomes Measurement Information Systems (PROMIS) component of CHOIR is an electronic patient 
survey, administered to all patients in the Stanford Pain Management Center. Approximately $40 \%$ of patients seen at the Stanford Pain Management Center successfully complete the CHOIR questionnaires. CHOIR collects longitudinal data using set measures at initial clinic visits, at fixed intervals thereafter and at follow-up treatments and has been used as a platform for prior pain research. ${ }^{27-29}$ In this study, all patients completed the questionnaires for their initial medical appointment at the Stanford Outpatient Pain Management Center.

\section{Measures}

\section{Perceived injustice}

Perceived injustice was measured with the Injustice Experience Questionnaire (IEQ). ${ }^{1}$ The IEQ asks respondents to rate how frequently they experience each of the 12 pain-related thoughts (e.g., "Nothing will ever make up for what I have gone through"). A total IEQ score is computed by summing the 12 items with higher scores reflecting higher levels of perceived injustice. The IEQ contains two correlated factors labeled "severity of loss/irreparability of loss" and "blame/ unfairness". The IEQ has shown to have good internal consistency and test-retest reliability. The coefficient alpha for the total IEQ in this study was 0.92 , which is consistent with previous studies. ${ }^{1}$

\section{Pain intensity}

Current pain intensity was measured using a Numeric Rating Scale (NRS) that operates on a $0-10$ scale with " 0 " being no pain and " 10 " being the worst pain imaginable. ${ }^{30}$ The NRS has been validated for specificity and use in chronic pain research. $^{30,31}$

\section{Depressive symptoms}

Within CHOIR, PROMIS is delivered as a computer-based survey that uses a computerized adaptive testing (CAT) approach based on item response theory (IRT) to allow for item-level responses, greater precision achieved through lowered standard error and a smaller set of questions with reduced sensitivity to population variability. ${ }^{32,33}$ The PROMIS depression item bank examines the frequency at which a person experiences the cognitive, affective and behavioral aspects of depression (e.g., "I felt depressed" and "I felt like I had nothing to look forward to"). Items are rated on a 6-point scale from "never" to "always", where a higher final score indicates more depressive symptoms. The recall period for the depression items was the past 7 days. PROMIS instruments are normed on the US population and are reported using $t$-scores with a mean of 50 and a standard deviation (SD) of
$10 .{ }^{34}$ The PROMIS depression item bank has demonstrated validity and consistency. ${ }^{35}$

\section{Pain behavior}

The PROMIS pain behavior item bank examines the frequency at which an individual engages in both communicative pain behavior (e.g., "When I was in pain I gasped") and protective pain behavior (e.g., "I limped because of pain"). Items are rated on a 6-point scale from "never" to "always", where a higher final score indicates more pain behavior. The recall period for the pain behavior items was the past 7 days. The PROMIS pain behavior item bank has demonstrated validity and consistency. ${ }^{36}$

\section{Opioid prescription}

Patients were asked to report all current opioid prescriptions, either electronically via CHOIR or verbally to medical staff. Opioid prescription was recorded as a binary variable with $0=$ no opioid prescription and $1=$ any opioid prescription.

\section{Patient characteristics}

All patients were asked to provide information on their age, sex, education level, marital status and pain type (e.g., musculoskeletal, disk, nerve).

\section{Data analysis}

Relationships among patient characteristics data and questionnaire data were analyzed using Pearson's correlations, chi-square analyses and independent samples $t$-test analyses, depending on whether the variables were ordinal or numerical. Mean and SD values were computed on all sample characteristics and questionnaire scores. $t$-Tests for independent samples were used to compare women and men, as well as individuals with and without an active opioid prescription on all study measures. These analyses were conducted using IBM SPSS Statistics version 21.0 (IBM Corporation, 2012).

A multiple mediation analysis was conducted to assess whether pain intensity, depressive symptoms or pain behavior mediated the association between perceived injustice and opioid prescription. By conducting a multiple mediation analysis, we were able to test the overall mediation effect for all mediators included in the model (total indirect effect) and to test the effects of each mediator separately (specific indirect effects). These specific indirect effects are used to determine whether the independent variable (IV) exerts its effect on the dependent variable (DV) through the mediator, while controlling for all other mediators in the model. ${ }^{37}$ 
The multiple mediation analysis was conducted using the SPSS macro (process) developed by Preacher and Hayes. . $^{37,38}$ Bootstrapping is a nonparametric procedure that is increasingly being used to test mediation (indirect) effects. It provides a way of circumventing power deficiencies of normal theory tests (e.g., Sobel) typically introduced by the non-normality in the sampling distribution. ${ }^{39-41}$ Bias-corrected (BC) $95 \%$ confidence intervals (CIs) were produced for each potential mediator and were used to test the significance of total and specific indirect (mediation) effects. Estimates of indirect effects were considered significant in the case zero was not included within the CIs. ${ }^{37,38}$ Bootstrap analyses were conducted using patient sex and age as covariates. Study variables were standardized before performing the mediation analysis.

\section{Ethical concerns}

Study procedures, which involved exclusively retrospective review of clinical data, were approved by the institutional review board (IRB) at the Stanford University School of Medicine. All participants provided written informed consent.

\section{Results}

\section{Sample demographic characteristics}

Demographic characteristics for the 344 patients included in this study are described in Table 1. Most of the study sample was married $(\mathrm{n}=184 ; 54 \%)$, with at least some college education $(\mathrm{n}=282 ; 82 \%)$, and composed of women $(\mathrm{n}=230$; $67 \%$ ). Mean age of the sample was 48 years and ranged from 18 to 85 years. Most patients in the sample reported nerve-related pain (49\%), pain due to unknown causes (31\%), musculoskeletal pain (23\%) and disk-related pain (21\%).

\section{Relationships among study variables}

Men and women did not differ in their perceived injustice scores $(t(342)=0.765, p=0.456)$, pain intensity scores $(t(342)=-0.022, p=0.938)$, depressive symptom scores $(t(342)=0.485, p=0.628)$ and pain behavior scores $(t=-1.75$, $p=0.08)$. Men and women also did not differ according to opioid prescription $\left(\chi^{2}=0.096, p=0.792\right)$. Analyses revealed that individuals with active opioid prescriptions differed significantly on all study variables (Table 2).

Pearson's product-moment correlations among the selfreport variables are presented in Table 3. Consistent with previous research, perceived injustice was significantly correlated with pain intensity, depressive symptoms and pain behavior. ${ }^{2}$ Results also revealed that pain intensity, depressive symptoms and pain behavior were significantly intercorrelated. Tests for multicollinearity indicated that a
Table I Sample demographic characteristics

\begin{tabular}{ll}
\hline Variables & $\mathbf{n}(\%)$ \\
\hline Sex & \\
Men & $114(33)$ \\
Women & $230(67)$ \\
Age, years & \\
I8-30 & $49(14)$ \\
$31-40$ & $70(20)$ \\
$41-50$ & $69(20)$ \\
$51-60$ & $88(26)$ \\
6I-70 & $48(14)$ \\
7 I-80+ & $18(5)$ \\
Marital status & \\
Separated/divorced & $21(6)$ \\
Cohabitating & $41(12)$ \\
Widowed & $83(24)$ \\
Married & $184(54)$ \\
Never married & $14(12)$ \\
Education & \\
No high school diploma & $32(9)$ \\
High school diploma or equivalent & $30(9)$ \\
Some college, no degree & $76(22)$ \\
Some university/associate's degree & $37(11)$ \\
Bachelor's degree & $88(25)$ \\
Graduate degree & $81(24)$ \\
Pain type & \\
Nerve & $169(49)$ \\
Musculoskeletal & $79(23)$ \\
Disk & $72(21)$ \\
Unknown cause & $107(31)$ \\
\hline Note:N-344. &
\end{tabular}

Note: $\mathrm{N}=344$.

Table 2 Results of $t$-tests comparing perceived injustice, pain intensity, depressive symptoms and pain behavior on opioid prescription

\begin{tabular}{llll}
\hline Variables & \multicolumn{2}{l}{ Active opioid prescription } & \multirow{2}{*}{-Test } \\
\cline { 2 - 3 } & No, M (SD) & Yes, M (SD) & \\
\hline Perceived injustice & $15.60($ II.40) & $20.17($ I0.5I) & $-3.82 * *$ \\
Pain intensity & $4.44(2.78)$ & $5.68(2.37)$ & $-3.26 * *$ \\
Depressive symptoms & $54.12(9.9)$ & $57.89(9.5)$ & $-3.15^{* *}$ \\
Pain behavior & $57.87(6.05)$ & $60.58(4.34)$ & $-3.69 * *$ \\
\hline
\end{tabular}

Notes: Mean scores and standard deviation for the study variables according to opioid prescription are shown. $t$-test, independent samples $t$-test. ${ }^{* *} p<0.00 \mathrm{I}$.

Abbreviations: $M$, mean; SD, standard deviation.

Table 3 Correlations among study variables

\begin{tabular}{llll}
\hline Variables & I & $\mathbf{2}$ & $\mathbf{3}$ \\
\hline I. Perceived injustice & & & \\
2. Pain intensity & $0.333^{* *}$ & & \\
3. Depressive symptoms & $0.656^{* *}$ & $0.308^{* *}$ & \\
4. Pain behavior & $0.374^{* *}$ & $0.522^{* *}$ & $0.403^{* *}$ \\
\hline
\end{tabular}

very low level of multicollinearity was present (pain intensity, tolerance $=0.717$ and variance inflation factor $(\mathrm{VIF})=1.395$; depressive symptoms, tolerance $=0.824$ and VIF $=1.213$; pain behavior, tolerance $=0.663$ and VIF $=1.509$ ). 


\section{Potential mediating role of pain intensity, depressive symptoms and pain behavior in the association between perceived injustice and opioid prescription}

The potential mediating role of pain intensity, depressive symptoms and pain behavior in the association between perceived injustice and opioid prescription was examined using a $\mathrm{BC}$ bootstrapped multiple mediation analysis (with $\mathrm{N}=5,000$ bootstrap re-samples). As shown in Table 4 and Figure 1, results of the multiple mediation analysis revealed that the direct effect of perceived injustice on opioid prescription (path c1) was not significant, suggesting potential mediation. The total (i.e., overall) indirect effect of pain intensity, depressive symptoms and pain behavior on the association between perceived injustice and opioid prescription was significant as the BC bootstrapped 95\% CI (95\% BC CI) did not include zero. All mediation analyses controlled for age and sex as covariates.

\section{Specific indirect effect (pain intensity)}

Results of the multiple mediation analysis revealed a significant effect of perceived injustice on pain intensity (path a1; $p<0.001)$. However, the direct effect of pain intensity on opioid prescription was nonsignificant (path b1; ns). Moreover, the indirect effect of pain intensity (path $\mathrm{a} 1 \times \mathrm{b} 1$ ) was not significant, as the $95 \%$ BC CI contained zero. Taken together, these results suggest that pain intensity did not mediate the association between perceived injustice and opioid prescription.

\section{Specific indirect effect (depressive symptoms)}

Results of the multiple mediation analysis revealed a significant effect of perceived injustice on depressive symptoms
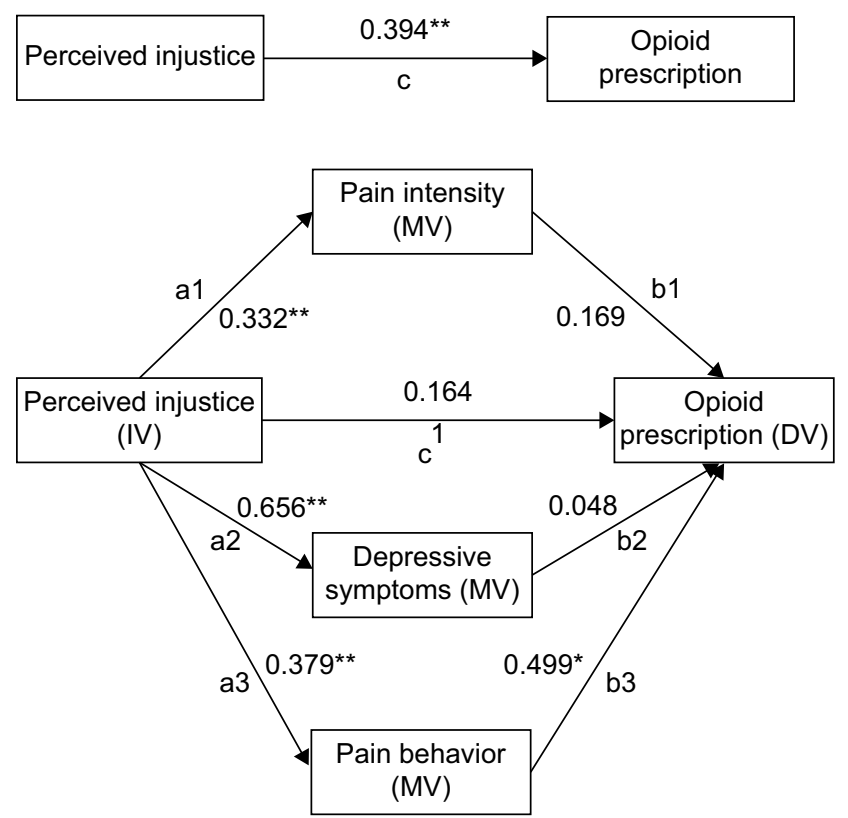

Figure I The mediating effect of pain intensity, depressive symptoms and pain behavior on the association between perceived injustice and opioid prescription. Notes: Analyses controlled for age and sex. Path coefficients are presented. ${ }^{*} p<$ $0.05 ; * * p<0.001$.

Abbreviations: IV, independant variable; DV, dependant variable; MV, mediating variable.

Table 4 Bootstrapped multiple mediation analysis testing the indirect effect of perceived injustice on opioid prescription through pain intensity, depressive symptoms and pain behavior

\begin{tabular}{|c|c|c|c|c|}
\hline Variables & Path coefficient & Bootstrap SE & $t$ & $95 \% \mathrm{BC} \mathrm{Cl}$ \\
\hline Path c & 0.394 & 0.124 & $3.172^{*}$ & \\
\hline Path cl & 0.164 & 0.168 & 0.974 & \\
\hline Path al & 0.332 & 0.051 & $6.497^{*}$ & \\
\hline Path a2 & 0.656 & $0.04 I$ & 15.986* & \\
\hline Path a3 & 0.379 & 0.050 & $7.573^{*}$ & \\
\hline Path bl & 0.169 & 0.153 & 1.110 & \\
\hline Path b2 & 0.048 & 0.181 & 0.271 & \\
\hline Path b3 & 0.499 & 0.239 & $2.092 * *$ & \\
\hline \multicolumn{5}{|l|}{ Specific indirect effects } \\
\hline $\mathrm{al} \times \mathrm{bl}$ & 0.057 & 0.053 & & $\mathrm{LL}=-0.0436, \mathrm{UL}=0.1686$ \\
\hline $\mathrm{a} 2 \times \mathrm{b} 2$ & 0.031 & 0.129 & & $\mathrm{LL}=-0.2187, \mathrm{UL}=0.2932$ \\
\hline $\mathrm{a} 3 \times \mathrm{b} 3$ & 0.189 & 0.122 & & $\mathrm{LL}=0.0122, \mathrm{UL}=0.4936$ \\
\hline \multicolumn{5}{|l|}{ Total indirect effects } \\
\hline $\mathrm{albl} \times \mathrm{a} 2 \mathrm{~b} 2 \times \mathrm{a} 3 \mathrm{~b} 3$ & 0.276 & 0.148 & & $\mathrm{LL}=0.0307, \mathrm{UL}=0.5767$ \\
\hline
\end{tabular}

Notes: Standardized path coefficients for the total and specific indirect effects are shown. Path c, total effect of perceived injustice on opioid prescription; path cl, direct effect of perceived injustice on opioid prescription; path al, effect of perceived injustice on pain intensity; path a2, effect of perceived injustice on depressive symptoms; path a3, effect of perceived injustice on pain behavior; path bl, direct effect of pain intensity on opioid prescription; path b2, direct effect of depressive symptoms on opioid prescription; path b3, direct effect of pain behavior on opioid prescription; path al $\times$ bl, indirect effect of perceived injustice on opioid prescription through pain intensity; path a2 $\times$ b2, indirect effect of perceived injustice on opioid prescription through depressive symptoms; path a3 $\times$ b3, indirect effect of perceived injustice on opioid prescription through pain behavior; path a $\mathrm{lbl} \times \mathrm{a} 2 \mathrm{~b} 2 \times \mathrm{a} 3 \mathrm{~b} 3$, total indirect effect of perceived injustice on opioid prescription through pain intensity, depressive symptoms and pain behavior. Path coefficients are based on 5,000 bootstraps for the indirect effect. LL and UL Cls were used to determine statistical significance of indirect effects. All analyses controlled for age and sex. ${ }^{*} p<0.001$; ${ }^{*} p<0.05$.

Abbreviations: $\mathrm{BC}$, bias corrected; $\mathrm{Cl}$, confidence interval; LL, lower limit; SE, standard error; UL, upper limit. 
(path a2; $p<0.001$ ). However, the direct effect of depressive symptoms on opioid prescription was nonsignificant (path b2; ns). Moreover, the indirect effect of depressive symptoms (path a $2 \times$ b2) was not significant, as the $95 \%$ BC CI contained zero. Taken together, these results suggest that depressive symptoms did not mediate the association between perceived injustice and opioid prescription.

\section{Specific indirect effect (pain behavior)}

Results of the multiple mediation analysis revealed a significant effect of perceived injustice on pain behavior (path a3; $p<0.001$ ) and a significant direct effect of pain behavior on opioid prescription (path $\mathrm{b} 3 ; p<0.05$ ). Results revealed that the indirect effect of pain behavior (path a $3 \times$ b3) was significant as the $95 \%$ BC CI did not include zero. Taken together, these results suggest that pain behavior mediated the association between perceived injustice and opioid prescription.

\section{Discussion}

The findings of this study add to a growing body of literature suggesting that perceived injustice is associated with numerous adverse pain-related outcomes in patients with chronic pain. The results of this study are consistent with previous studies that demonstrated that higher levels of perceived injustice are associated with opioid prescription in individuals with whiplash and musculoskeletal injury., ${ }^{3,4}$ This study extends past research by using a large sample of individuals seeking specialized pain care with various types of chronic pain and therefore allows us to generalize our findings to a wider population of chronic pain patients. Furthermore, this study advances previous work by examining the potential mediating role of pain intensity, depressive symptoms and pain behavior in the association between perceived injustice and opioid prescription for chronic pain.

Results did not support the proposed mediation model with pain intensity as mediator of the relationship between perceived injustice and opioid prescription for chronic pain. Our findings indicated that patients with higher levels of perceived injustice reported higher levels of pain intensity, which is consistent with the results of previous studies. ${ }^{1,4,8,42}$ We also found that high pain intensity was associated with opioid prescription; however, the results of our mediation analysis revealed that pain intensity did not mediate the association between perceived injustice and opioid prescription. Although significantly correlated with pain intensity, the strength of the association between perceived injustice and pain intensity was relatively weaker than the correlations between perceived injustice and depressive symptoms and pain behavior. It has been suggested that prescribers may be more likely to respond to patients' behavioral manifestations of pain, to distress related to pain and to the impact on functional activities than to reports of pain severity and more objective physical findings when they decide to prescribe opioids for chronic pain. ${ }^{20}$

Our findings also did not support the mediation model with depressive symptoms as a mediator of the relationship between perceived injustice and opioid prescription. In accordance with previous research, results showed that perceived injustice was strongly associated with depressive symptoms ${ }^{1,6-11}$ and that patients who were prescribed opioids reported higher symptoms of depression. ${ }^{12-14}$ However, mediation analyses revealed that depressive symptoms did not mediate the association between perceived injustice and opioid prescription. Although depressive symptoms did not explain the association between injustice and opioid prescription, future research is needed to examine whether depressive symptoms are responsible, at least in part, for continued or long-term opioid therapy in patients with perceptions of injustice. It has been suggested that psychiatric comorbidity increases the rate of long-term opioid use and misuse through inappropriate self-medication of distress or depressive feelings. ${ }^{43-45}$

In accordance with previous research, perceived injustice was significantly associated with self-reported pain behavior. These findings parallel those of Sullivan et al, ${ }^{17}$ who found that high levels of perceived injustice were associated with heightened display of pain behavior in individuals with whiplash injury. Importantly, in our study, results demonstrated that self-reported pain behavior mediated the association between perceived injustice and opioid prescription in patients with chronic pain. In fact, although the overall indirect effect reached significance, closer examination of the specific indirect effects revealed that only pain behavior emerged as a mediator of the relationship between perceived injustice and opioid prescription. These data extend previous research that identified that pain behavior as a possible mechanism linked perceived injustice and pain-related disability $^{17}$ and provide the first support for pain behavior as a mediator of the relationship between perceived injustice and opioid prescription. The results of this study highlight the importance of pain behavior as an integral component of the pain experience. ${ }^{46}$ Indeed, the current focus on the sensory and psychological dimensions of the pain experience may have limited theoretical advance and may impede the development of effective treatment approaches for individuals with chronic pain. 
The communications model of pain explains that individuals often communicate their pain experience through the display of various behaviors, which are then interpreted and decoded by observers. ${ }^{19}$ As mentioned previously, observers place great emphasis on the credibility of these behaviors and view them as reliable indicators of the intensity of a person's pain. ${ }^{47-50}$ In fact, clinicians tend to assign greater weight to behavioral expressions of pain, rather than self-reports, when judging the location and severity of pain..$^{20,51}$ However, pain behavior has been shown to only modestly associate with pain intensity and tends to persist over time independently of patients' symptoms of pain. ${ }^{52-54}$ It is possible that individuals who view their pain in terms of injustice display more pain behavior as a means of communicating the intensity of their suffering and losses, which inadvertently increases their likelihood of being prescribed opioids for chronic pain. Longitudinal studies are needed to identify the nature of this relationship and the temporal sequencing of the effect of perceived injustice and pain behavior on opioid prescription in this population.

The findings of this study have implications for the treatment of patients with chronic pain. Because pain behavior is largely determined by cognitive and affective factors, intervention techniques specifically designed to target the determinants of pain behavior might yield better outcomes than interventions aimed simply at reducing pain intensity or depressive symptoms. Several investigators have suggested that intervention approaches such as validation, acceptance-commitment therapy and forgiveness might be useful in yielding reductions in perceived injustice. ${ }^{4,55,56}$ If used in conjunction with such interventions, innovative tools such as video feedback interventions, graded activity interventions or exposure may help increase patient's behavioral awareness and reduce maladaptive pain behavior. ${ }^{46,57}$ However, this study was cross-sectional and did not investigate the impact of such interventions on perceived injustice and pain behavior.

Optimal management of perceived injustice and pain behavior also requires consideration by the clinicians who are involved in the treatment of patients with pain conditions and particularly those who may be called upon to make judgments about prescribing opioids for chronic pain. Clinicians involved in the treatment of pain have long been aware of the behavioral displays that accompany the pain experience and are prominent components of pain-related disability. In the absence of empirical evidence concerning the meaning of pain behavior, clinicians have generally relied on their own intuitive inferences. ${ }^{46}$ However, with the current epidemic of opioid prescription use and abuse, ${ }^{58-60}$ prescribers must be prepared with the skills to intervene with less aggressive treatment options. Efforts must be made to increase prescribers' understanding that pain behavior is more strongly determined by psychological factors than pain severity and how prescribing actions may be influenced by patients' pain behavior. More specifically, prescribers need to be made aware that expressions of pain behavior do not necessarily reflect a more severe pain condition and that opioids might not be the best approach to manage psychological aspects of pain.

In a clinical setting, the assessment of pain behavior is useful in many ways. ${ }^{36}$ First, it can provide information regarding the intensity and causes of pain. Second, it can provide insights into a person's attempts to cope or manage their pain. Third, it can reveal maladaptive coping efforts that can be targeted in interventions. In fact, it has been suggested that pain behavior provides a behavioral marker of enhanced risk for the development of chronic pain and disability. ${ }^{61}$ Although direct observation provides the most objective approach to assessing pain behavior, it has limitations (e.g., need for ongoing observer training, costs, intrusiveness) that limit its utility in clinical settings. An interesting alternative strategy for measuring pain behavior, which was used in this study, is self-report. This provides a cost-efficient and low-burden option for experimenters and clinicians. Researchers have emphasized that self-report of pain behavior also assesses the pain experience, incorporating beliefs, attitudes and perceptions, and therefore provides a more subjective, yet valid reflection of the patient's experience. ${ }^{61}$ To effectively gather both subjective and objective information, researchers suggest using a variety of complementary assessment tools..$^{52}$

There are limitations to this study that must be considered when interpreting our findings. As noted earlier, the cross-sectional nature of this study design precludes any firm conclusions regarding the directionality of associations between study variables. Although results of mediation analyses imply potential directional influences among variables, it cannot be determined whether perceived injustice is a precursor of pain behavior and whether displays of pain behavior are precursors of opioid prescription. This type of longitudinal research is required in order to confirm our findings. The generalizability of this study may be limited due to the relative homogeneity of this sample. Our sample included patients seeking specialty pain care who may have higher levels of pain-related disability than patients seeking treatment in primary care or in community chronic pain samples. Thus, we must be cautious in generalizing about the factors influencing opioid prescription beyond the population of patients referred to tertiary pain treatment 
centers. Moreover, pain behavior and depressive symptoms were assessed solely on the basis of patient's self-reports. However, both PROMIS item banks have good evidence supporting their reliability and validity. ${ }^{35,36}$ As mentioned earlier, future studies should use, if possible, multiple measurement methods (e.g., patients' self-reports, physician ratings, videotaping) in order to assess pain behavior. Finally, data regarding opioid prescription were binary and did not provide any information regarding the dose, the use or the misuse of opioid prescriptions. Future research might examine the influence of injustice and pain behavior on prescribed opioid dosage and possible misuse.

\section{Conclusion}

The findings of this research provide interesting insight into the mechanisms by which perceived injustice may impact on opioid prescription. The key finding of this study is that pain behavior mediated the association between perceived injustice and opioid prescription. Pain intensity and depressive symptoms did not explain the association between perceived injustice and opioid prescription. These findings highlight how some individuals who are high in perceived injustice may use pain behavior to emphasize the magnitude of their suffering and, in turn, be more likely to be prescribed more aggressive treatments, such as opioids for chronic pain. Future research will be needed to examine whether the relationships observed in this study can be replicated longitudinally. Advances in these areas may improve early identification of patients at risk for opioid prescription and may mitigate risk for adverse outcomes. Research is also needed to identify other possible mediators of the relationship between perceived injustice and opioid prescription for chronic pain.

\section{Acknowledgments}

We acknowledge funding support from the National Institutes of Health (NIH) National Center for Complementary and Integrative Health (NCCIH) P01AT006651 (SCM) and P01AT006651S1 (SCM and BDD); NCCIH R01AT008561 (BDD and SCM); NIH National Institute on Drug Abuse (NIDA) K24DA029262 (SCM); NIH Pain Consortium HHSN271201200728P (SCM); the Chris Redlich Pain Research Endowment (SCM) and the Quebec Pain Research Network (JSC). The abstract of this paper was presented at the 20th Annual McGill Pain Day as a poster presentation talk with interim findings. The poster's abstract was published in "Poster Abstracts" in the Journal of Pain: DOI: http://dx.doi. org/10.1016/j.jpain.2016.01.425.

\section{Disclosure}

The authors report no conflicts of interest in this work.

\section{References}

1. Sullivan MJL, Adams H, Horan S, Maher D, Boland D, Gross R. The role of perceived injustice in the experience of chronic pain and disability: scale development and validation. J Occup Rehabil. 2008; 18(3):249-261

2. Sullivan MJL, Yakobov E, Scott W, Tait R. Perceived injustice and adverse recovery outcomes. Psychol Inj Law. 2014;7(4):325-334.

3. Scott W, Trost Z, Bernier E, Sullivan MJL. Anger differentially mediates the relationship between perceived injustice and chronic pain outcomes. Pain. 2013;154(9):1691-1698.

4. Scott W, Trost Z, Milioto M, Sullivan MJL. Further validation of a measure of injury-related injustice perceptions to identify risk for occupational disability: a prospective study of individuals with whiplash injury. J Occup Rehabil. 2013;23(4):557-565.

5. Mikula G, Scherer KR, Athenstaedt U. The role of injustice in the elicitation of differential emotional reactions. Pers Soc Psychol Bull. 1998;24(7):769-783.

6. Scott W, Sullivan M. Perceived injustice moderates the relationship between pain and depressive symptoms among individuals with persistent musculoskeletal pain. Pain Res Manag. 2012;17(5):335-340.

7. Sullivan MJL, Thibault P, Simmonds MJ, Milioto M, Cantin A-P, Velly AM. Pain, perceived injustice and the persistence of post-traumatic stress symptoms during the course of rehabilitation for whiplash injuries. Pain. 2009;145(3):325-331.

8. Trost Z, Agtarap S, Scott W, et al. Perceived injustice after traumatic injury: associations with pain, psychological distress, and quality of life outcomes 12 months after injury. Rehabil Psychol. 2015;60(3):213-221.

9. Rodero B, Luciano JV, Montero-Marín J, et al. Perceived injustice in fibromyalgia: psychometric characteristics of the Injustice Experience Questionnaire and relationship with pain catastrophising and pain acceptance. J Psychosom Res. 2012;73(2):86-91.

10. Scott W, Trost Z, Milioto M, Sullivan MJL. Barriers to change in depressive symptoms after multidisciplinary rehabilitation for whiplash. Clin J Pain. 2015;31(2):145-151.

11. Scott W, Milioto M, Trost Z, Sullivan MJL. The relationship between perceived injustice and the working alliance: a cross-sectional study of patients with persistent pain attending multidisciplinary rehabilitation. Disabil Rehabil. 2016;38(24):2365-2373.

12. Sullivan MD, Edlund MJ, Fan M-Y, Devries A, Braden JB, Martin BC. Risks for possible and probable opioid misuse among recipients of chronic opioid therapy in commercial and medicaid insurance plans: The TROUP Study. Pain. 2010;150(2):332-339.

13. Braden JB, Sullivan MD, Ray GT, et al. Trends in long-term opioid therapy for noncancer pain among persons with a history of depression. Gen Hosp Psychiatry. 2009;31(6):564-570.

14. Cowan DT, Wilson-Barnett J, Griffiths P, Allan LG. A survey of chronic noncancer pain patients prescribed opioid analgesics. Pain Med. 2003;4(4):340-351.

15. Katon W, Sullivan M, Walker E. Medical symptoms without identified pathology: relationship to psychiatric disorders, childhood and adult trauma, and personality traits. Ann Intern Med. 2001;134(9 pt 2):917-925.

16. Katon WJ. Epidemiology and treatment of depression in patients with chronic medical illness. Dialogues Clin Neurosci. 2011;13(1):7-23.

17. Sullivan MJL, Davidson N, Garfinkel B, Siriapaipant N, Scott W. Perceived injustice is associated with heightened pain behavior and disability in individuals with whiplash injuries. Psychol Inj Law. 2009; 2(3-4):238-247.

18. Sullivan MJL, Scott W, Trost Z. Perceived injustice: a risk factor for problematic pain outcomes. Clin J Pain. 2012;28(6):484-488.

19. Hadjistavropoulos T, Craig KD. A theoretical framework for understanding self-report and observational measures of pain: a communications model. Behav Res Ther. 2002;40(5):551-570. 
20. Turk DC, Okifuji A. What factors affect physicians' decisions to prescribe opioids for chronic noncancer pain patients? Clin J Pain. 1997; 13(4):330.

21. Boscarino JA, Hoffman SN, Han JJ. Opioid-use disorder among patients on long-term opioid therapy: impact of final DSM-5 diagnostic criteria on prevalence and correlates. Subst Abuse Rehabil. 2015;6:83-91.

22. Sullivan MD, Ballantyne JC. What are we treating with long-term opioid therapy? Arch Intern Med. 2012;172(5):433-434.

23. Jamison RN, Serraillier J, Michna E. Assessment and treatment of abuse risk in opioid prescribing for chronic pain. Pain Res Treat. 2011;2011(2):1-12.

24. Turk DC, Swanson KS, Gatchel RJ. Predicting opioid misuse by chronic pain patients: a systematic review and literature synthesis. Clin J Pain 2008;24(6):497-508.

25. Kircher S, Zacny J,Apfelbaum SM, et al. Understanding and treating opioid addiction in a patient with cancer pain. J Pain. 2011;12(10):1025-1031.

26. Kao M, Weber S, Cook K, et al. (258) Stanford-NIH Pain Registry: open source platform for large-scale longitudinal assessment and tracking of modern patient-reported outcomes. J Pain. 2014;15(4 suppl):S40.

27. Sturgeon JA, Dixon EA, Darnall BD, Mackey SC. Contributions of physical function and satisfaction with social roles to emotional distress in chronic pain: a Collaborative Health Outcomes Information Registry (CHOIR) study. J Occup Rehabil. 2015;156(12):2627-2633.

28. Sturgeon JA, Darnall BD, Kao M-CJ, Mackey SC. Physical and psychological correlates of fatigue and physical function: a Collaborative Health Outcomes Information Registry (CHOIR) Study. J Pain. 2015;16(3):291.e1-298.e1.

29. Sturgeon JA, Carriere JS, Kao M-CJ, Rico T, Darnall BD, Mackey SC. Social disruption mediates the relationship between perceived injustice and anger in chronic pain: a Collaborative Health Outcomes Information Registry Study. Ann Behav Med. 2016;50(6):802-812.

30. Farrar JT, Young JP, LaMoreaux L, Werth JL, Poole RM. Clinical importance of changes in chronic pain intensity measured on an 11-point numerical pain rating scale. Pain. 2001;94(2):149-158.

31. Hjermstad MJ, Fayers PM, Haugen DF, et al; European Palliative Care Research Collaborative (EPCRC). Studies comparing Numerical Rating Scales, Verbal Rating Scales, and Visual Analogue Scales for assessment of pain intensity in adults: a systematic literature review. $J$ Pain Symptom Manage. 2011;41(6):1073-1093.

32. Fries JF, Bruce B, Cella D. The promise of PROMIS: using item response theory to improve assessment of patient-reported outcomes. Clin Exp Rheumatol. 2005;23(5 suppl 39):S53-S57.

33. Hahn EA, DeVellis RF, Bode RK, et al. Measuring social health in the patient-reported outcomes measurement information system (PROMIS) item bank development and testing. Qual Life Res. 2010;19(7):1035-1044.

34. Cella D, Yount S, Rothrock N, et al; PROMIS Cooperative Group. The patient-reported outcomes measurement information system (PROMIS): progress of an NIH roadmap cooperative group during its first two years. Med Care. 2007;45(5 suppl 1):S3-S11.

35. Pilkonis PA, Choi SW, Reise SP, et al. Item banks for measuring emotional distress from the patient-reported outcomes measurement information system (PROMIS $®$ ): depression, anxiety, and anger. Assessment. 2011;18(3):263-283.

36. Revicki DA, Chen W-H, Harnam N, et al. Development and psychometric analysis of the PROMIS pain behavior item bank. Pain. 2009; 146(1):158-169.

37. Preacher KJ, Hayes AF. SPSS and SAS procedures for estimating indirect effects in simple mediation models. Behav Res Methods Instrum Comput. 2004;36(4):717-731.

38. Preacher KJ, Hayes AF. Asymptotic and resampling strategies for assessing and comparing indirect effects in multiple mediator models. Behav Res Methods. 2008;40(3):879-891.
39. Hayes AF. Beyond Baron and Kenny: statistical mediation analysis in the new millennium. Commun Monogr. 2009;76(4):408-420.

40. Mackinnon DP, Lockwood CM, Hoffman JM, West SG, Sheets V. A comparison of methods to test mediation and other intervening variable effects. Psychol Methods. 2002;7(1):83-104.

41. Shrout PE, Bolger N. Mediation in experimental and nonexperimental studies: new procedures and recommendations. Psychol Methods. 2002;7(4):422-445.

42. Yakobov E, Scott W, Stanish W, Dunbar M, Richardson G, Sullivan M. The role of perceived injustice in the prediction of pain and function after total knee arthroplasty. Pain. 2014;155(10):2040-2046.

43. Wasan AD, Butler SF, Budman SH, Benoit C, Fernandez K, Jamison RN. Psychiatric history and psychologic adjustment as risk factors for aberrant drug-related behavior among patients with chronic pain. Clin J Pain. 2007;23(4):307-315.

44. Jamison RN, Butler SF, Budman SH, Edwards RR, Wasan AD. Gender differences in risk factors for aberrant prescription opioid use. J Pain. 2010;11(4):312-320.

45. Geisser ME, Cano A, Foran H. Psychometric properties of the mood and anxiety symptom questionnaire in patients with chronic pain. Clin J Pain. 2006;22(1):1-9.

46. Sullivan MJL. Toward a biopsychomotor conceptualization of pain: implications for research and intervention. Clin J Pain. 2008;24(4): 281-290.

47. Prkachin KM, Currie NA, Craig KD. Judging nonverbal expressions of pain. Can J Behav Sci. 1983;15(4):409-421.

48. Martel MO, Thibault P, Roy C, Catchlove R, Sullivan MJL. Contextual determinants of pain judgments. Pain. 2008;139(3):562-568.

49. Martel MO, Thibault P, Sullivan MJL. Judgments about pain intensity and pain genuineness: the role of pain behavior and judgmental heuristics. J Pain. 2011;12(4):468-475.

50. Craig KD, Prkachin KM, Grunau RVE. The Facial Expression of Pain. New York City, NY: Guilford Press; 1992

51. Hill ML, Craig KD. Detecting deception in facial expressions of pain: accuracy and training. Clin J Pain. 2004;20(6):415.

52. McCahon S, Strong J, Sharry R, Cramond T. Self-report and pain behavior among patients with chronic pain. Clin J Pain. 2005;21(3):223.

53. Labus JS, Keefe FJ, Jensen MP. Self-reports of pain intensity and direct observations of pain behavior: when are they correlated? Pain. 2003;102(1-2):109-124.

54. Martel MO, Thibault P, Sullivan MJL. The persistence of pain behaviors in patients with chronic back pain is independent of pain and psychological factors. Pain. 2010;151(2):330-336.

55. Sullivan MJL, Adams H, Martel M-O, Scott W, Wideman T. Catastrophizing and perceived injustice: risk factors for the transition to chronicity after whiplash injury. Spine. 2011;36(25 suppl):S244-S249.

56. Martel M-E, Dionne F, Scott W. The mediating role of pain acceptance in the relation between perceived injustice and chronic pain outcomes in a community sample. Clin J Pain. Epub 2016 Aug 12:1.

57. Keefe FJ, Williams DA, Smith SJ. Assessment of Pain Behaviors. New York, NY: Guilford; 2001:170-187.

58. Centers for Disease Control and Prevention (CDC). CDC grand rounds: prescription drug overdoses-a US epidemic. MMWR Morb Mortal Wkly Rep. 2012;61(1):10-13.

59. Manchikanti L, Helm S, Fellows B, et al. Opioid epidemic in the United States. Pain Physician. 2012;15(3 suppl):ES9-ES38.

60. Maxwell JC. The prescription drug epidemic in the United States: a perfect storm. Drug Alcohol Rev. 2011;30(3):264-270.

61. Jensen MP. Validity of self-report and observation measures. In: Jensen TS, Turner JA, Wiesenfeld-Hallin Z, editors. Proceedings of the 8th World Congress on Pain. Progress in Pain Research and Management. Vol. 8. Seattle, WA: IASP Press. 1997:637-661. 
The Journal of Pain Research is an international, peer reviewed, open access, online journal that welcomes laboratory and clinical findings in the fields of pain research and the prevention and management of pain. Original research, reviews, symposium reports, hypothesis formation and commentaries are all considered for publication.

Submit your manuscript here: https://www.dovepress.com/journal-of-pain-research-journal

The manuscript management system is completely online and includes a very quick and fair peer-review system, which is all easy to use. Visit http://www.dovepress.com/testimonials.php to read real quotes from published authors. 